\title{
Results from ISOCAM Deep Surveys: An Answer on the AGN Contribution to the Cosmic Infrared Background
}

\author{
Hervé Aussel \\ Institute for Astronomy, University of Hawaii, 2680 Woodlawn Drive, \\ Honolulu, Hawaii, 96822, USA
}

\begin{abstract}
The amount of energy contributed by type-2 AGNs to the Cosmic Infrared Background (CIB) has recently been the subject of some debate, since models have shown it could be responsible for up to half. If this were the case, this contribution should be taken carefully into account before using the combined $\mathrm{CIB}$ and COB to derive the density of metals in the universe. I argue here that an observational answer to this problem comes from ISOCAM deep surveys performed at $15 \mu \mathrm{m}$, that do resolve into discrete sources the bulk of the CIB at $140 \mu \mathrm{m}$. Studies of the X-ray properties of these sources allow us to assess whether or not they are dominated by AGNs, and to derive that the type- 2 AGNs contribution to the CIB is not greater than $20 \%$.
\end{abstract}

\section{Introduction}

The Extragalactic Background Light (EBL) is the cumulative emission of all extragalactic objects along the line of sight. It is a very powerful tool for the study of galaxy evolution (Partridge \& Peebles 1967): if it is produced by star light, it can be related to the present content of metals in the universe almost independently of the assumed cosmology (Cowie 1988; Bernstein, Weedman \& Madore 2002). Starlight clearly dominates in the optical region (between 0.1 and $5 \mu \mathrm{m})$ where the EBL is often referred to as the Cosmic Optical Background (COB). However, measurements of the EBL in the X-ray and infrared/submillimeter domain have prompted new questions and generated some debate.

First, a far-infrared/sub-millimeter EBL has been discovered in the FIRAS data by Puget et al. (1996), at a very significant level. The values of this Cosmic Infrared Background (CIB) have been subsequently refined and extended to the DIRBE data down to $100 \mu \mathrm{m}$ by Hauser et al. (1998) and Lagache et al. (2000) (see Figure 1). While in the local universe, about one third of the star light is absorbed by dust and reemited in the infrared (Soifer \& Neugebauer 1991), this fraction is inverted in the EBL: between half and 2/3 of its integrated energy is emitted above $10 \mu \mathrm{m}$. This indicates that the amount of star light reprocessed by dust must increase with redshift, and that the infrared part of the EBL has to be taken into account to derive the metal content of the universe.

Second, the EBL has been measured in the X-ray domain (Cosmic X-ray Background: CXB) at a much lower level than the COB and the CIB (Giacconi et al. 1962). Deep ROSAT surveys have resolved $80 \%$ of the CXB in the range 
0.5-2 keV, and subsequent follow-up studies have shown that it is produced by type-1 AGNs. However, such sources cannot explain the peak of the CXB at $30 \mathrm{keV}$. Comastri et al. (1995) have suggested that the high energy part of the CXB is due to a population of heavily obscured type-2 AGNs. Such a population has been confirmed by recent XMM/Newton (Hasinger et al. 2001) and Chandra surveys (Mushotsky et al. 2000) in the $2-10 \mathrm{keV}$ range. While type-1 AGNs do not emit a significant fraction of their bolometric energy above $10 \mu \mathrm{m}$, such a population of type-2 AGNs has important implications on the nature of the CIB. Indeed, models of the CXB predict that an important fraction of the CIB is due to accretion in AGNs, from $20 \%$ up to $50 \%$ (Almaini, Lawrence \& Boyle 1999). If this is the case, the contribution of this accretion-dominated emission has to be subtracted from the CIB before using it in conjunction with the COB to derive any conclusion on galaxy evolution and metal production in the universe.

It is therefore crucial to determine precisely the nature of the sources producing the bulk of the CIB at $200 \mu \mathrm{m}$. This can only be done by resolving the CIB into discrete sources and determining whether they are dominated by star formation or AGN emission. Unfortunately, neither IRAS nor ISOPHOT were sensitive enough to resolve a significant fraction of the CIB directly (see lower limits from counts on Figure 1). However, I will show we now have compelling evidence that mid-IR deep extragalactic surveys performed with ISOCAM at $15 \mu \mathrm{m}$ have indeed resolved the CIB between $100 \mu \mathrm{m}$ and $200 \mu \mathrm{m}$, and that we now have observational clues on the fraction of its energy originating from type- 2 AGNs.

I will first review some basic properties of the EBL and discuss briefly the clues obtained as to its nature from sub-millimeter surveys. I will then review the results obtained in the mid-infrared (i.e. about $10 \mu \mathrm{m}$ ) that lead to the resolution of the bulk of the CIB: that mid-infrared extragalactic deep surveys performed by ISOCAM have resolved the CIB into discrete sources not only at the wavelength they were performed, but also in the far-infrared. I will then discuss the nature of the sources producing the CIB and show that it is mostly emitted by star formation.

\section{The Extragalactic Background Light (EBL)}

Predicted by Partridge \& Peebles (1967), the EBL is the cumulative emission of all extragalactic objects. At frequency $\nu$, its intensity is given by:

$$
I(\nu)=\int_{0}^{+\infty} S(\nu) d N
$$

where $d N$ is the number of extragalactic sources observed by unit areas with flux between $S$ and $S+d S$ at frequency $\nu$. We can rewrite (1) by assuming that the differential number count $d N$ follows a power law in $S$, i.e $d N \propto S^{-\gamma}$ and we have

$$
I(\nu)=\int_{0}^{+\infty} S(\nu)^{1-\gamma} d S
$$

It is clear from (2) that the slope $\gamma$ of the differential number counts has to be lower than 2 in order for the background integral to converge, and that its intensity will be dominated by the region where the counts have a slope of $\gamma=2$. 


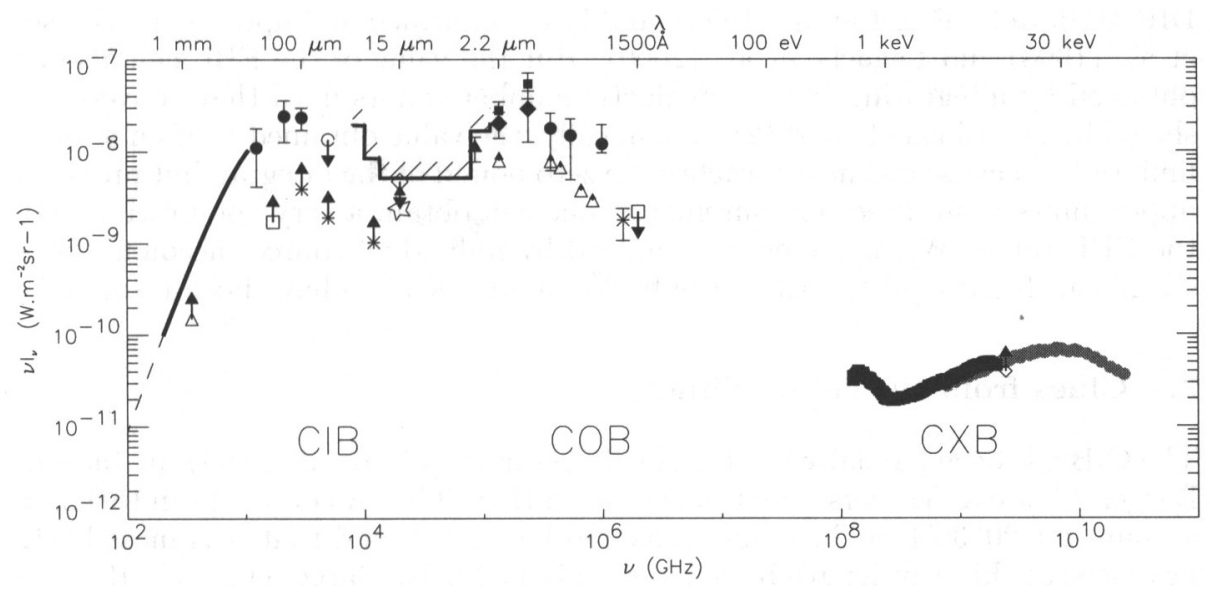

Figure 1. Spectral energy distribution of the Extragalactic Background Light (EBL). Measurements are in solid symbols, upper and lower limits in open symbols. Cosmic Infrared Background (CIB): solid line: best fit of the FIRAS detection by Lagache et al. (1999) - circles: DIRBE detections from Lagache et al. (2000) - open circle: upper limit from $60 \mu \mathrm{m}$ IRAS maps (Mirville-Deschenes, Lagache \& Puget 2002) - hatched region: upper limit from $\gamma$-rays absorption (Stanev \& Franceschini 1998) - diamond: upper limit $\gamma$-rays absorption at $15 \mu \mathrm{m}$ (Renault et al. 2000) - open star: ISOCAM number counts lower limit (Elbaz et al. 2002) - stars: IRAS number counts lower limits (Lonsdale et al. 1990) - square : ISOPHOT $170 \mu \mathrm{m}$ lower limit (Dole et al. 2001) - Triangle : SCUBA $850 \mu \mathrm{m}$ counts lower limit (Barger, Cowie \& Sanders 1999). For the Cosmic Optical Background (COB): triangle: $3.5 \mu \mathrm{m}$ DIRBE detection (Gordjian, Wright \& Chary 2000) - diamond: (DIRBE/2MASS) detection (Wright 2001) - square: (DIRBE/2MASS) detection (Cambrésy et al. 2001) - circles: Bernstein, Freedman \& Madore (2002) detection with HST- open triangle: HDF number counts (Pozzetti et al. 1998) - star: FOCA detection (Armand, Milliard \& Deharveng 1994). open square : Martin, Hurwitz \& Bowyer (1991) upper limit. Cosmic X-ray Background (CXB) black squares: ASCA/ROSAT measurements (Gilli, Salvati \& Hasinger 2001) - grey circles: HEAO1 measurements (Gruber, Matteson \& Peterson 1999) - open diamond: Chandra number counts (Mushotsky et al. 2000) 
Figure 1 displays the current estimates for the spectral energy distribution (SED) of the EBL. Such estimates can be direct measurements of an isotropic diffuse signal by experiments lacking angular resolution. This is the case in the far-infrared and sub-millimeter where the EBL was detected in the FIRAS and DIRBE data by Puget et al. (1996), and later confirmed and updated by Hauser et al. (1998) and Lagache et al. (2000). But the value of the EBL can also be obtained by integrating the extragalactic number counts until they converge as shown by equations (1) and (2). Technically, the value obtained is often a lower limit only, because one never reaches the zero bound of the integral, but provided upper limits from direct measurements, one can obtain a very good estimate of the EBL value. When the energy emitted by individual sources accounts for a significant fraction of the EBL, the background is said to have been resolved.

\section{Clues from the sub-millimeter}

The CIB has been partially resolved by deep surveys obtained at $850 \mu \mathrm{m}$. Indeed, Barger, Cowie \& Sanders (1999) have shown that SCUBA counts down to $2 \mathrm{mJy}$ account for $20-30 \%$ of the value measured by FIRAS and that it is most likely produced at this wavelength by sources of about $1 \mathrm{mJy}$. Barger et al. (2001) have compared Chandra and SCUBA observations of SSA13. Their X-ray sample consists of 20 sources and is complete at a level of $3 \times 10^{-15} \mathrm{erg} \mathrm{s}^{-1} \mathrm{~cm}^{-2}$, where between $58 \%$ and $89 \%$ of the $2-10 \mathrm{keV} \mathrm{CXB}$ is resolved. Only one source is recovered in their $850 \mu \mathrm{m}$ map with an average sensitivity of $1.5 \mathrm{mJy}$. They deduce that the contribution of type-2 AGNs producing the CXB to the CIB at $850 \mu \mathrm{m}$ is the range $9-13 \%$.

A similar conclusion is reached by Severgini et al. (2000) who have studied a sample of hard X-ray sources observed by both Beppo-SAX and SCUBA. They also point out that most of the SCUBA population is dominated by star formation or Compton thick AGNs that contribute less than $6 \%$ of the CXB.

Both these studies point toward a low contribution of type-2 AGNs to the CIB. However, they can only make conclusions about the CIB at $850 \mu \mathrm{m}$. Unfortunately, the bulk of the CIB is observed between $100 \mu \mathrm{m}$ and $250 \mu \mathrm{m}$ : while SCUBA counts are dominated by luminous sources at redshift above 2 , the CIB is produced by sources at lower redshifts where type-2 AGNs may have a different contribution. The definitive answer can only be obtained by resolving the bulk of the CIB.

\section{ISOCAM resolves the EBL at $15 \mu \mathrm{m}$}

ISOCAM (Cesarsky et al. 1999), the mid-infrared imager on board the ISO satellite has conducted many extragalactic surveys at $15 \mu \mathrm{m}$, at various depth and area coverage. The result of these observations are shown in Figure 2 from Elbaz et al. (2000), where the differential extragalactic number counts, normalized to the euclidean slope $(\gamma=2.5$ in equation 2$)$, are plotted against the observed flux. Five important remarks can be made on Figure 2: 


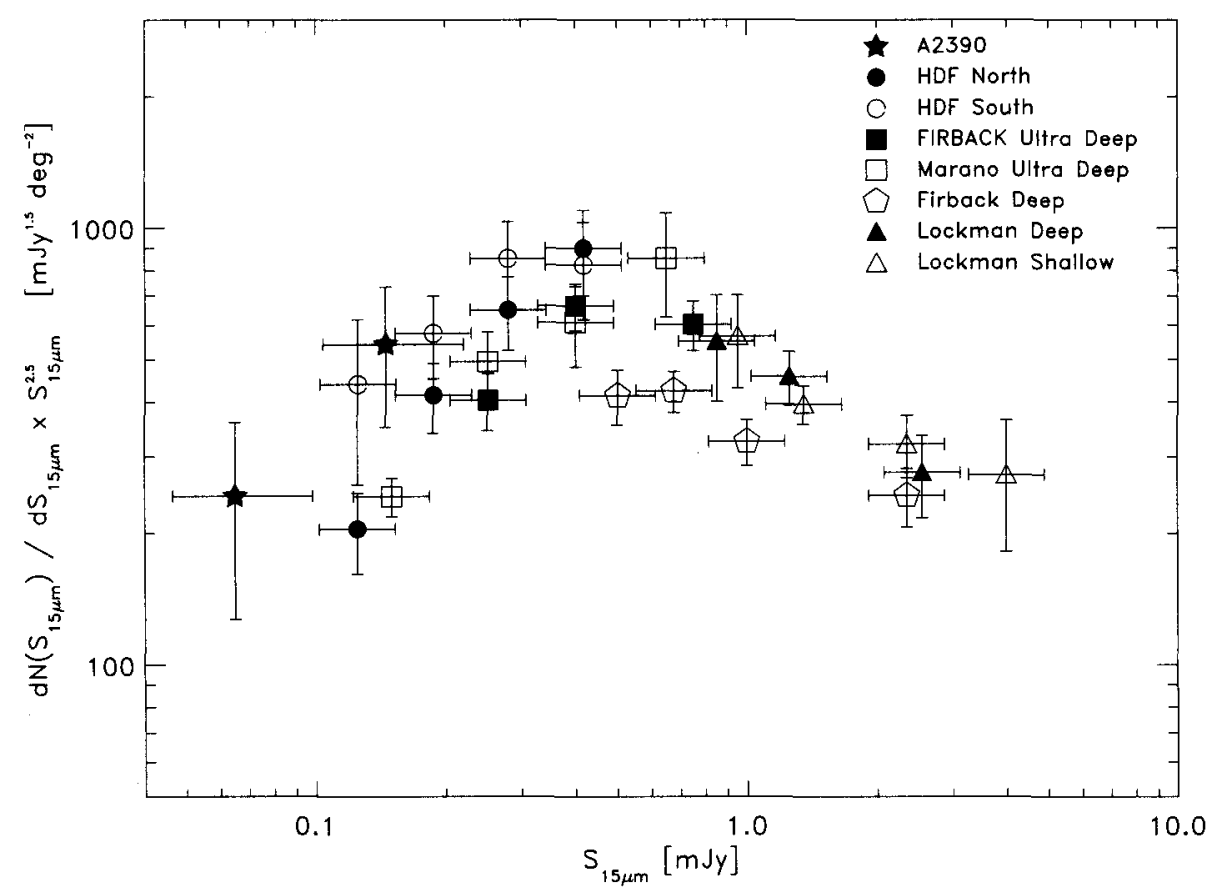

Figure 2. Extragalactic number counts at $15 \mu \mathrm{m}$ from ISOCAM deep surveys, normalized to the euclidean slope, after Elbaz et al. (2000).

1) With respect to IRAS, ISOCAM has extended the depth of mid-infrared extragalactic surveys by more than 3 orders of magnitude, from $200 \mathrm{mJy}$ in the IRAS Faint Source Survey (Lonsdale et al. 1990) to $50 \mu \mathrm{Jy}$ in the deepest areas.

2) All the number counts obtained on different Northern and Southern fields agree.

3) At $400 \mu \mathrm{Jy}$, the number of sources observed per square degree is $\sim 3000$, a factor of 10 in excess of the predictions of models without evolution (Franceschini et al. 2002).

4) The slope of the counts at high flux levels is super-euclidean with a slope of the differential counts of $\gamma=3 \pm 0.1$. This cannot be explained alone by kcorrection effects in the $15 \mu \mathrm{m}$ filter, and strong evolution has to be advocated to reproduce the counts. $S^{-1.6}$

5) The slope of the counts at $S_{\nu}<400 \mu \mathrm{Jy}$ is under-euclidean, with $d N(S) \propto$

Point 5 is especially important, because it means that the number counts are converging below $400 \mu \mathrm{Jy}$. This means that we can obtain a reliable estimate of the value of the CIB at $15 \mu \mathrm{m}$ by integrating the ISOCAM and IRAS counts, using equations 1 and 2. This exercise has been done by Elbaz et al. (2002) where a value of $2.4 \pm 0.5 \mathrm{nW} \mathrm{m}^{-2} \mathrm{sr}^{-1}$ has been derived. 
As noted in $\S 2$, this result would only constitute a lower value of the CIB at $15 \mu \mathrm{m}$ if the counts were to steepen again at lower flux. Fortunately, we can obtain reliable upper limits for the value mid-IR CIB by studying the absorption of $\gamma$-ray photons emitted by blazars by the mid-IR photon radiation field between the source and the observer through the reaction $\gamma+\gamma \rightarrow e^{+}+e^{-}$. Renault et al. (2001) derive a very good upper limit for the CIB at $15 \mu \mathrm{m}$ of $4.7 n \mathrm{Wm}^{-2} \mathrm{sr}^{-1}$. This upper limit is very close to the lower limit derived from ISOCAM counts.

Both upper and lower limits are also in good agreement with the predicted value by Franceschini et al. (2001) of $3.3 \mathrm{nW} \mathrm{m}^{-2} \mathrm{sr}^{-1}$. Adopting this figure, we deduce that ISOCAM deep surveys have resolved $73 \%$ of the $15 \mu \mathrm{m}$ CIB.

\section{ISOCAM resolves the bulk of the CIB}

Admittedly, the title of this section is provocative. Indeed, ISOCAM observations were performed at $15 \mu \mathrm{m}$, not between $100 \mu \mathrm{m}$ and $250 \mu \mathrm{m}$ where the bulk of the CIB is observed. I will, however, argue that provided reasonable assumptions on the SED of the galaxies detected in ISOCAM surveys, it is possible to compute their contributions in the FIR, and show that they account for a large fraction of the CIB.

A first computation was performed by Aussel, Elbaz \& Cesarsky (1999), assuming that all ISOCAM detected sources were Ultra Luminous Infrared Galaxies (ULIRGs) with a SED similar to the one of Arp 220 and with a median redshift of $z \sim 0.75$. With such simple assumptions, the CIB at $140 \mu \mathrm{m}$ produced by the ISOCAM detected galaxies would be of the order of $250 \mathrm{nW} \mathrm{m}^{-2} \mathrm{sr}^{-1}$, far above the actual measurement of $24.2 \mathrm{nW} \mathrm{m}^{-2} \mathrm{sr}^{-1}$.

A more precise determination has been made by Elbaz et al. (2002), using the ISOCAM observations of the Hubble Deep Field (HDF) (Aussel et al. 1999). Let us note first from Figure 2 that the HDF samples the flux region where the counts flatten. Hence, according to Equation 2, the $15 \mu \mathrm{m}$ EBL should be dominated by the sources observed at such a flux. This is indeed the case, with $48 \%$ of the $15 \mu \mathrm{m} \mathrm{CIB} \mathrm{beeing} \mathrm{resolved} \mathrm{between} 100 \mu \mathrm{Jy}$ and $500 \mu \mathrm{Jy}$, the flux range sampled by the ISO-HDF.

The HDF region is extremely interesting, because a nearly complete redshift survey has been obtained down to $R=23.5$ by Cohen et al. (2000), and because it has also been observed at radio wavelengths (Richards 2000; Garret et al. 2000 ) and in X-rays (Brandt et al. 2000). In this region, the ISOCAM sample consists of 41 galaxies, complete down to $100 \mu \mathrm{Jy}$. All galaxies but one have a redshift in the Cohen et al. (2000) catalog. It is therefore possible to compute the mid-IR luminosity of the ISOCAM galaxies: the main difficulty is the $\mathrm{k}$ correction of the observed $15 \mu \mathrm{m}$ flux, given the complicated shape of the SED of galaxies in the mid-IR region and its variation from object to object.

To overcome this problem, Elbaz et al. (2002) use a library of templates for the whole infrared spectrum of galaxies calibrated with local samples, together with the fact that the mid-IR luminosity is an excellent tracer of the total bolometric luminosity of galaxies (Spinoglio et al. 1995). Given the observed luminosity without the $k$-correction term (i.e. the luminosity at $\lambda_{o b s}=$ $\left.\lambda_{\text {rest }} /(1+z)=15 \mu \mathrm{m}\right)$, they compute a range of possible SEDs for the galaxies, their IR luminosities and their contributions at $140 \mu \mathrm{m}$. They find that the con- 
tribution of the ISOCAM galaxies detected in the HDF at $15 \mu \mathrm{m}$ contribute to the $140 \mu \mathrm{m}$ CIB is $16.0 \pm 4.6 \mathrm{nW} \mathrm{m}^{-2} \mathrm{sr}^{-1}$, where the quoted error comes from the spread in possible SEDs for each galaxy. This constitutes at least $30 \%$ of the measured value, and possibly for all of it. Hence, the ISOCAM deep surveys do resolve the bulk of the CIB. In comparison, ISOPHOT deep surveys only resolve $4 \%$ of the CIB at $170 \mu \mathrm{m}$ (Dole et al. 2001), due to the lack of sensitivity of the instrument.

It is interesting to note that in the Elbaz et al. (2002) computations, ULIRGs only contribute $16 \%$ of the $15 \mu \mathrm{m}$ CIB, but $36 \%$ of the $140 \mu \mathrm{m}$ CIB, while LIRGs $\left(L \in\left[10^{11} L_{\odot}, 10^{12} L_{\odot}\right]\right)$ represent respectively $44 \%$ and $48 \%$ of the $15 \mu \mathrm{m}$ and $140 \mu \mathrm{m}$ CIB. This is a first indication that the contribution of type-2 AGNs to the bulk of the CIB might be low, since Genzel et al. (1998) show that the central black hole is the dominant source of power only in the most luminous sources (but see Antonucci in these proceedings for a critique of this work). Indeed, Elbaz et al. (2002) estimate the contribution of AGNs in the HDF to $\sim 19 \%$ of the $15 \mu \mathrm{m}$ EBL and only $4 \%$ to the $140 \mu \mathrm{m}$ EBL due to the flatter SED of these objects.

Therefore, it appears from this work that the CIB is largely dominated by star formation. An independent test of this assumption can be made : 21 of the 41 ISOCAM galaxies are detected at $1.4 \mathrm{GHz}$ in the HDF by Richards (2000) and Garrett et al. (2000). Using the model of Condon (1992), we can compute the star formation rate (SFR) in these galaxies from their radio flux, and compare it to the one derived from the infrared luminosities: an excellent agreement is found, with a slope of 1 and a dispersion compatible with the photometric errors (Aussel 2002). Moreover, the ISOCAM galaxies that are not detected in the radio have, from their IR-inferred SFR, 1.4 GHz fluxes below the detection limits of both the VLA and Westerbrok observations.

\section{The AGN contribution to the EBL from ISOCAM surveys}

It has been pointed out many times during this conference that the only means to detect a dust embedded AGN is by observing in the hard X-ray domain. Hence, a good constraint on the contribution of type-2 AGNs to the CIB can be obtained by following up the ISOCAM sources with XMM/Newton and Chandra. Fadda et al. (2002) present such a study in the HDF and the Lockman Hole fields.

The Lockman Hole field has been observed by both XMM/Newton and ISO$\mathrm{CAM}$ in a region of 218 square arc minutes. The X-ray observation reach a sensitivity of $1.4 \times 10^{-15} \mathrm{erg} \mathrm{s}^{-1} \mathrm{~cm}^{-2}$ in the $2-10 \mathrm{keV}$ band and $2.4 \times 10^{-15} \mathrm{erg} \mathrm{s}^{-1} \mathrm{~cm}^{-2}$ in the $2-10 \mathrm{keV}$ band, respectively resolving $80 \%$ and $60 \%$ of the CXB in these bands. At $15 \mu \mathrm{m}$, the ISOCAM observation probes the $500 \mu \mathrm{Jy}-2 \mathrm{mJy}$ flux range (see Figure 2) and resolves $23 \%$ of the CIB at this wavelength (Elbaz et al. 2002). Twenty-four of the $76 \mathrm{X}$-ray sources are detected in the mid-IR, with the fraction of $15 \mu \mathrm{m}$ detection rising with X spectrum hardness: from $30 \%$ in the $0.5-2 \mathrm{keV}$ band to $63 \%$ in the $5-10 \mathrm{keV}$ band. This is a confirmation that the hard CXB is produced by type-2 AGNs. On the other hand, only $10 \%$ of the ISOCAM sources are detected in the X-ray.

Fadda et al. (2002) combine these results fromon the Lockman Hole with the cross-correlation of the Chandra $1 \mathrm{Ms}$ exposure (Brandt et al. 2001) and 
the ISOCAM $15 \mu \mathrm{m}$ catalog of the HDF (Aussel et al. 1999). Using the median spectral index between X-ray and mid-IR of the sources detected in both fields, and assuming that all the sources detected in $X$-ray are $A G N$-dominated, they infer a contribution of type- 2 AGNs to the $15 \mu \mathrm{m}$ CIB of $17 \pm 2 \%$ and note that this figure is also an upper limit to their contribution to the whole CIB, since the SED of these objects peaks at $20 \mu \mathrm{m}$.

This conclusion has been refined by Alexander et al. (2002) using the $1 \mathrm{Ms}$ Chandra and ISOCAM data on the HDF, combined with the spectroscopic catalog of Cohen et al. (2000). They note that up to $100 \%$ of the X-ray sources that have been spectroscopically classified as emission line galaxies (ELGs) are detected at $15 \mu \mathrm{m}$, while the mid-IR detection rate of absorption line galaxies and AGN-dominated spectra are at the $20 \%$ level. Though none of these ELGs are detected in the hard Chandra band (2-8 keV) (probably because Chandra is less sensitive than XMM in the hard band), they perform a statistical analysis of their spectral slope by stacking all of the ELG X-ray detections and deduce a photon index $\Gamma \simeq 2$. This is an higher value than the photon index derived for the population of optically faint $(I \geq 24)$ X-ray detections thought to be type-2 AGNs for which $\Gamma \simeq 1.3$. Also, the range of soft $\mathrm{X}$-ray band luminosities of these sources is compatible with those of local starbursts like M 82 and NGC 3256.

This led Alexander et al. (2002) to the conclusion that most of the X-ray emission of the $15 \mu \mathrm{m}$ detected sources does not come from an AGN, but from star formation. Therefore, the assumption used by Fadda et al. (2002) that all $\mathrm{X}$-Ray sources are dominated by an AGN is much too strong, and the numbers they derive for the contribution of AGNs to the $15 \mu \mathrm{m}$ and $140 \mu \mathrm{m}$ CIB are upper limits.

\section{Conclusion}

The ISOCAM deep surveys at $15 \mu \mathrm{m}$ have led to the resolution into discrete sources of the CIB both in the mid- and far-IR. From the infrared, radio and Xray properties of the sources producing the CIB, it appears that at least $85 \%$ of it is due to star formation, with an upper limit of type-2 AGN's contribution of $15 \%$ at $100 \mu \mathrm{m}$ to $250 \mu \mathrm{m}$ where the bulk of the CIB is emitted. In comparison, type- 1 AGNs represent only about $2 \%$ of the CIB at $15 \mu \mathrm{m}$ and do not contribute noticeably to the whole CIB because of their very warm SED (Matute et al. 2002).

It should be noted that this conclusion is reached mainly by using the HDF data set that is composed of a flux-limited complete sample of $4115 \mu \mathrm{m}$ detected sources with measured redshifts. Hopefully, the statistics will improve as more spectroscopic followups of ISOCAM deep fields are undertaken, in the Lockman Hole, the Marano Field and the HDF-South (see Figure 2). Together, these fields represent a sample of more than 500 sources that will allow improved measurements. From early unpublished results on these fields, it appears that the conclusions presented here will not change drastically, and that the high value predicted by some models (Almaini, Lawrence \& Boyle 1999) is ruled out.

In the near future, SIRTF with its MIPS instrument will perform surveys at $24 \mu \mathrm{m}$ that will resolve the CIB between $200 \mu \mathrm{m}$ and $400 \mu \mathrm{m}$ in the same way that ISOCAM did. Both CAM and MIPS results will be tested by FIRST/Hershel 
that will be the first satellite to be in a position to resolve directly the CIB between $60 \mu \mathrm{m}$ and $500 \mu \mathrm{m}$.

\section{Acknowledgments}

I thank the organizers for their invitation to this extremely interesting conference that has prompted the further investigations of the IR/X-ray properties of ISOCAM galaxies presented in $\S 5$. I thank my collaborators D. Alexander, D. Elbaz and D. Fadda for their inputs. This research is supported by a Fellowship of the James Clerk Maxwell Telescope.

\section{References}

Alexander, D. M. et al. 2002, ApJ, 568, L85

Almaini, O., Lawrence, A. \& Boyle, B. 1999, MNRAS, 305, 59

Armand, N. A., Milliard, B. \& Deharveng, J. M. 1994, A\&A, 284, 12

Aussel, H. 2002, Ap\&SS, 281, 441

Aussel, H. et al. 1999, A\&A, 342, 313

Aussel, H., Elbaz, D. \& Cesarsky C. J. 1999, Ap\&SS, 266, 307

Barger, A. J., Cowie, L. L. \& Sanders, D. B. 1999, ApJ, 518, L5

Barger, A. J. et al. 2001, AJ, 121, 662

Bernstein, R. A., Freedman, W. L. \& Madore, B. F. 2002, ApJ, 571, 107

Brandt, W. N. et al. 2001, AJ, 122, 1

Cambrésy, L. et al. 2001, A\&A, 555, 563

Cesarsky, C. J. et al. 1996, A\&A, 315, L32

Comastri, A. et al. 1995, A\&A, 296, 1

Condon, J. J. 1992, ARA\&A, 30, 575

Cohen, J. G. et al. 2000, ApJ, 538, 29

Cowie, L. L. 1988, in Proc. of the NATO ASI Vol. 240, The Post-Recombination Universe, ed. N. Kaiser \& A. N. Lasenby (Dortrecht: Kluwer), 1

Dole, H. et al. 2001, A\&A, 372, 364

Elbaz, D. et al. 1999, A\&A, 351, L37

Elbaz, D. et al. 2002, A\&A, 384, 848

Garrett, M. A. et al. 2000, A\&A, 361, L41

Giacconi R. et al. 1962, Phys.Rev.Lett, 9, 439

Gilli, R., Salvati, M. \& Hasinger, G. 2001, A\&A, 366, 407

Gordjian, V., Wright, E. L. \& Chary, R. R. 2000, ApJ, 536, 550

Gruber, D. E., Matteson, J. L. \& Peterson, L. E. 1999, ApJ, 520, 124

Fadda, D. et al. 2002, A\&A, 383, 838

Franceschini, A. et al. 2001, A\&A, 378, 1

Genzel, R. et al., 1998 ApJ, 498, 579

Hauser, M. G. et al. 1998, ApJ, 508, 25 
Lagache, G. et al. 1999, A\&A, 344, 322

Lagache, G. et al. 2000, A\&A, 354, 247

Lonsdale, C. J. et al. 1990, ApJ, 358, 60

Martin, C, Hurwitz, M. \& Bowyer, S. 1991, A\&A, 379, 549

Matute, I. et al. 2002, MNRAS, 332, L11

Mirville-Deschene, M. A. \& Lagache, G. \& Puget J.-L., A\&A, in press, (astro$\mathrm{ph} / 0207312$ )

Mushotsky, R. F. et al. 2000, Nature, 404, 459

Partridge, R. B. \& Peebles, P. J. E. 1967, ApJ, 148, 377

Pozzetti, L. et al. 1998, MNRAS, 298, 1133

Puget, J.-L., et al. 1996, A\&A, 308, L5

Renault, C. et al. 2001, A\&A, 371, 771

Richards, E. A. 2000, ApJ, 533, 611

Severgnini, P. et al. 2000, A\&A, 360, 457

Spinoglio, L. et al. 1995, ApJ, 453, 616

Soifer, B. T. \& Neugebauer, G. 1991, AJ, 101, 354

Stanev, T \& Franceschini, A. 1998, ApJ, 494, L59

Wright, E. L. 2001, ApJ, 553, 538 